\title{
Conocimientos, actitudes y prácticas sobre una alimentación saludable en adolescentes de dos Instituciones Educativas Públicas, Lima Este-2018
}

\section{Knowledge, attitudes and practices on healthy eating in adolescents of two Public Educational Institutions, Lima East-2018}

\section{Yanina Fustamante Ticlla', Giovanna Mirian Quispe Nuñoncca²}

\begin{abstract}
RESUMEN
Objetivo: Determinar el nivel de conocimientos, actitudes y prácticas sobre una alimentación saludable en los adolescentes de dos instituciones Educativas. Metodología: Diseño no experimental, de corte trasversal y de tipo descriptivo. La población estuvo conformada por todos los estudiantes del 1ro a 5 to grado del nivel secundario. La muestra estuvo conformada por 589 estudiantes. El instrumento fue un cuestionario de 42 preguntas sobre conocimientos, actitudes y prácticas. Resultados: Se encontró que el $57 \%$ de los adolescentes tiene un nivel de conocimiento regular, el $83.4 \%$ presenta una actitud favorable y el $78.2 \%$ de los adolescentes presentó una práctica inadecuada en alimentación saludable. Conclusiones: Más de la mitad de los adolescentes tiene un nivel de conocimiento regular. Por otro lado, se encontró una actitud favorable para optar por una alimentación saludable, aunque la mayor proporción no la practica adecuadamente.
\end{abstract}

Palabras claves: Pausa activa, Salud ocupacional, Clima laboral, Clima organizacional.

\begin{abstract}
Objective: Determine the level of knowledge, attitudes and practices on healthy eating in adolescents from two educational institutions. Methodology: Is non-experimental design, cross-sectional, and descriptive type. The population was made up of all the students of the 1st and 5th grade of the secondary level. The sample consisted of 589 students. The instrument was an applied questionnaire of 42 questions, knowledge, attitudes and practices. Results: It was found that $57 \%$ of adolescents have a regular level of knowledge, $83.4 \%$ have a favorable attitude and $78.2 \%$ of adolescents presented an inadequate practice in healthy eating. Conclusions: More than half of adolescents have a level of regular knowledge. On the other hand, it was found that they presented a favorable attitude in opting for a healthy diet. However, the largest proportion do not practice it adequately.
\end{abstract}

Keywords: Knowledge, attitudes, practices.

\footnotetext{
${ }^{1}$ Lic. Nutrición, Clínica Good Hope, Lima, Perú.

${ }^{2}$ Lic. Nutrición, Universidad Peruana Unión, Lima, Perú.
} 


\section{INTRODUCCIÓN}

La adolescencia es un periodo importante de la vida que da inicio a un cambio de caracteres físicos, psicológicos y fisiológicos entre la infancia y la edad adulta. Definitivamente, adquirir conocimientos sobre alimentación saludable en la adolescencia es muy importante, como base para la generación de hábitos alimenticios, pues asegura la satisfacción de las necesidades nutricionales, un adecuado aporte de energía y nutrientes, evitando carencias o excesos que puedan ser perjudiciales para la salud (Mondalgo, 2016).

Justamente, esta etapa se caracteriza por la búsqueda y afianzamiento de una personalidad definida $y$, en ese proceso, la necesidad de aceptación y pertenencia les conduce a ciertas prácticas inadecuadas, pero populares para verse "bellas". En ese contexto, practican patrones de alimentación definidos por las preferencias a determinadas comidas y alimentos que influyen en la aceptación de su imagen corporal, lo que conlleva a adquirir practicas alimentarias que podrían conducirlos a una alimentación desequilibrada en el afán de asemejarse a un prototipo de belleza (Delbino, 2013).

Las prácticas alimentarias inadecuadas de los adolescentes se tornan peligrosas porque, en la mayoría de los casos, se mantienen a lo largo de la vida adulta, conllevando a factores de riesgo para la salud. Diversos estudios demuestran, además, la enorme influencia de los medios publicitarios, que impregnan en ellos creencias totalmente erradas en temas de alimentación. Dicho impacto mediático también es considerado uno de los principales causantes del incremento de la obesidad en el mundo, ya que su alto nivel de atracción conduce al sedentarismo y a la ingesta de alimentos ricos en azúcares y grasas (Donayre, 2016).

De hecho, el fundamento para que los adolescentes practiquen una alimentación armoniosa, equilibrada y sana radica en que el organismo necesita cubrir sus necesidades biológicas básicas, y tener un óptimo desarrollo corporal, para lograr un buen rendimiento intelectual, físico, psicológico para desarrollarse socialmente, y evitar problemas posteriores de salud. Dada las circunstancias negativas que la industria "alimenticia" impone, surge la necesidad de mejorar y conocer los conocimientos, prácticas y actitudes en cuanto a la alimentación en los adolescentes de esta investigación para plantear futuros programas educativos.
La importancia de esta investigación titulada "Prevalencia de conocimientos, actitudes y prácticas sobre el consumo de una alimentación balanceada en adolescentes de dos Instituciones Educativas Públicas, Lima-2019" radica en que los resultados contribuirán a que los estudiantes mejoren sus hábitos alimenticios, a través de una alimentación saludable mediante programas de educación nutricional que orienten a mejorar y/o cambiar los comportamientos negativos y adoptar conductas saludables en cuanto a la frecuencia y cantidad de consumo de alimentos de acuerdo con las necesidades nutricionales del adolescente. Además, servirá de base para la realización de otros estudios similares.

\section{METODOLOGÍA}

Esta investigación es de diseño no experimental, de corte trasversal y de tipo descriptivo. La población de estudio estuvo conformada por los estudiantes de 1ro a 5to año de secundaria de las Instituciones Educativas San Luis María de Monfort y El Amauta. Se aplicó un cuestionario que contenía preguntas de conocimientos, actitudes y prácticas sobre una alimentación saludable.

\section{Muestra}

La muestra estuvo conformada por 589 estudiantes del colegio San Luis María de Monfort y El Amauta. La selección de la muestra se realizó mediante el muestreo no probabilístico de tipo intencional. (Hernández et al. 2010).

\section{Instrumento}

El instrumento tiene como finalidad recopilar datos sobre los conocimientos, actitudes y prácticas sobre alimentación saludable de los adolescentes. Este cuestionario fue elaborado según los criterios propuestos por la Organización de las Naciones Unidas para la Alimentación y Agricultura, (Delbino, 2013).

El instrumento fue elaborado para marcar y está dividido en 4 secciones y comprendido por 42 ítems ( 7 datos demográficos, 12 de conocimiento, 11 de actitudes y 12 de prácticas). El instrumento fue validado por juicio de expertos para ser puesto a prueba de fiabilidad aplicando una prueba piloto respectivamente. 
Conocimientos: El tipo de respuesta final por cada pregunta era dicotómica (Verdadero $=1$, Falso $=0)$. El valor final del cuestionario fue evaluado como: bajo (0-4), regular (5-8) y alto (9-12).

Actitudes: El tipo de respuesta final por cada pregunta era polinómico (Totalmente de acuerdo $=5$, De acuerdo $=3$, En desacuerdo $=2$, Totalmente en desacuerdo=1). El valor final del cuestionario fue evaluado como: desfavorable (122) y favorable (23-44).

Prácticas: El tipo de respuesta final por cada pregunta era polinómico (siempre $=4$, casi siempre $=3$, a veces $=2$, nunca $=1$ ). El valor final del cuestionario fue evaluado como: inadecuado (1-24) y Adecuado (25-48).

Para la prueba de fiabilidad se aplicó una prueba piloto a una muestra de 50 personas con características similares a la muestra objetivo.
La fiabilidad se determinó a través del análisis de Kuder-Richardson formula 20 para la variable de conocimientos, y Alfa de Crombach para las variables de actitudes y prácticas. El instrumento fue validado por alfa de Crombach 0.77.

\section{RESULTADOS}

En la tabla 1 se observa que el $54.2 \%$ de los estudiantes es de sexo masculino. Respecto a la edad, se observa que el $54.3 \%$ oscila entre los 12 a 14 años. En cuanto al nivel educativo, el 23.1\% está en primer grado, siguiendo con $21.1 \%$ de cuarto grado. Respecto al lugar de procedencia, un $77.1 \%$ proviene de la costa. En cuanto a los colegios, el $55.9 \%$ estudia en El Amauta. Finalmente, en cuanto al grado de instrucción, la mayoría de padres señaló el nivel secundario.

Tabla 1

Distribución de las características sociodemográficas.

\begin{tabular}{|c|c|c|}
\hline Variables & $\mathbf{N}$ & $\%$ \\
\hline \multicolumn{3}{|l|}{ Sexo } \\
\hline Femenino & 270 & $45.8 \%$ \\
\hline Masculino & 319 & $54.2 \%$ \\
\hline \multicolumn{3}{|l|}{ Edad } \\
\hline 12 a14 años & 320 & $54.3 \%$ \\
\hline 15 a 17 años & 269 & $45.7 \%$ \\
\hline \multicolumn{3}{|l|}{ Grado de estudio } \\
\hline Primero & 136 & $23.1 \%$ \\
\hline Segundo & 122 & $20.7 \%$ \\
\hline Tercero & 110 & $18.7 \%$ \\
\hline Cuarto & 124 & $21.1 \%$ \\
\hline Quinto & 97 & $16.5 \%$ \\
\hline \multicolumn{3}{|l|}{ Lugar de procedencia } \\
\hline Costa & 454 & $77.1 \%$ \\
\hline Sierra & 64 & $10.9 \%$ \\
\hline Selva & 44 & $7.5 \%$ \\
\hline Extranjero & 27 & $4.6 \%$ \\
\hline \multicolumn{3}{|l|}{ Colegio } \\
\hline El Amauta & 329 & $55.9 \%$ \\
\hline San Luis de María de Monfort & 260 & $44.1 \%$ \\
\hline \multicolumn{3}{|l|}{ Grado de instrucción (Mamá) } \\
\hline Sin estudio & 14 & $2.4 \%$ \\
\hline Primaria & 102 & $17.3 \%$ \\
\hline Secundaria & 336 & $57.0 \%$ \\
\hline Superior & 137 & $23.3 \%$ \\
\hline \multicolumn{3}{|l|}{ Grado de instrucción (Papá) } \\
\hline Sin estudio & 12 & $2.0 \%$ \\
\hline Primaria & 56 & $9.5 \%$ \\
\hline Secundaria & 372 & $63.2 \%$ \\
\hline Superior & 149 & $25.3 \%$ \\
\hline Total & 589 & $100.0 \%$ \\
\hline
\end{tabular}


Como se puede observar en la tabla 2, la mayoría de los estudiantes encuestados posee un nivel de conocimiento regular, resultados similares encontrados por Delbino (2013), quien evidenció que los niveles de conocimiento en los estudiantes son regulares en un $54 \%$ y más de la mitad conoce sobre la alimentación saludable. Asimismo, Cruz (2014), en Ecuador, encontró que el $92.6 \%$ de los encuestados demostró un nivel de conocimiento adecuado sobre alimentación saludable, aunque en este estudio se señala que solo el $27.9 \%$ recibe sesiones educativas sobre el tema. Como se puede observar, la proporción de los participantes que demostró un nivel de conocimiento adecuado es mayor a los que recibe sesiones educativas sobre el tema. Este resultado podría deberse a diferentes factores tales como la metodología aplicada, la misma población estudiada y el nivel de conocimiento de los padres de familia. Por otro lado, en el estudio que realizó Delbino (2013), en Argentina, se observa que los alumnos tienen un previo conocimiento influenciado por la familia en un $60 \%$ mientras que en el colegio en un $32 \%$. En cambio, en nuestro estudio este resultado podría haber sido influenciado por continuas capacitaciones que reciben tanto los estudiantes como profesores y padres de familia por el centro de salud aledaño.

También en nuestro estudio se encontró que el $88.5 \%$ de los adolescentes presenta un nivel alto de conocimiento en cuanto a la pregunta ¿Qué es una alimentación saludable? (ver tabla 3), Resultados parecidos encontrados por Rodríguez,et al. (2016) quienes, en su estudio realizado en Brasil, encontraron que el $95.3 \%$ de los encuestados respondió adecuadamente a la pregunta similar formulada en nuestro estudio.

No obstante, Baltodano,et al (2016), en un estudio realizado en Nicaragua, encontraron un nivel de conocimiento deficiente en la población de los dos colegios encuestados en un $18.32 \%$ y $27.77 \%$, respectivamente formando un $46.55 \%$. Los investigadores mencionaron que en los colegios existe un vacío de conocimientos sobre alimentación saludable el cual se ve evidenciado en sus respuestas. Por ello, han planteado realizar capacitaciones permanentes para mejorar sus conocimientos. También Suárez, et al. (2014), en la ciudad de Mérida-Venezuela, encontró que el $62.5 \%$ de la población de estudio presentó un nivel de conocimiento bajo sobre el término de alimentación. Estos resultados, según los investigadores se verían reflejados por el nivel de conocimiento de los padres sobre temas relacionados a alimentación saludable. Además de ello, el $55.7 \%$ de los adolescentes encuestados mencionó no haber recibido información relacionada en cuanto a una educación nutricional.

Así mismo, Mondalgo (2013) en su estudio que realizó en Lima-Perú, evidenció que el 95\% presentó un nivel de conocimiento bajo antes de aplicar su programa educativo. Estos resultados coinciden con los de Bin,et al,(2017), en el estudio que realizó en las ciudades de Selangor y Malacca en Arabia Saudita, en la que encontraron que solo el $16.5 \%$ de los encuestados tenía un nivel de conocimiento nutricional "bueno". Asimismo, Al-Yateem y Rossiter (2017) realizaron un estudio en Sharjah; donde encontraron que el $86 \%$ de los estudiantes presentaba un nivel bajo en conocimiento nutricional. Mencionaron que los resultados podrían deberse a la falta de capacitación para los educadores en cuanto a la promoción de estilos de vida saludable, así como a la falta de estrategias educativas que impliquen un trabajo multidisciplinario en la institución educativa. Hay un punto de concordancia entre los resultados de los diferentes autores y es el nivel de conocimiento sobre la alimentación saludable en los adolescentes.

Tabla 2

Nivel de conocimiento sobre alimentación saludable.

\begin{tabular}{lll}
$\begin{array}{ll}\text { Conocimientos sobre alimentación } \\
\text { saludable }\end{array}$ & $\mathrm{N}$ & $\%$ \\
\hline Bajo & 61 & 10.4 \\
Regular & 336 & 57.0 \\
Alto & 192 & 32.6 \\
Total & 589 & 100.0 \\
\hline
\end{tabular}


En la tabla 3 se encontró que la mayor parte de la muestra de los adolescentes presenta una actitud favorable. Los resultados encontrados en este estudio se asemejan a los encontrados por Suarez et al. (2014), donde se evidenciaron que el $83.3 \%$ de los adolescentes está dispuesto a tener una alimentación saludable y recibir capacitaciones en cuanto a los temas alimentación y nutrición. Asimismo, el $94.5 \%$ opina que las comidas rápidas no son beneficiosas para la salud. Los investigadores refieren que, a pesar de que los adolescentes tienen un adecuado conocimiento en este tema como factor de riesgo, consumen frecuentemente este tipo de alimentos. De igual manera, en el estudio que realizó Delbino (2013), encontró que casi el $100 \%$ de los estudiantes presentó un interés adecuado por comer saludablemente. Asimismo, los investigadores Bin et al (2017), encontraron que el $67.1 \%$ de los encuestados presentó una actitud favorable al igual que Abraham et al. (2017), en su estudio encontraron que el $65 \%$ de los adolescentes no demostró preferencias hacia los alimentos pocos saludables. Si bien en el estudio de Delbino (2013) la mayor proporción presentó una actitud positiva por los alimentos saludables; a pesar de ello el $90 \%$ de los estudiantes tiene una inclinación por alimentos poco saludables como snacks, facturas (pastelería fina), hamburguesas, panchos (hot dog), pizza, golosinas, etc.

Por el contario, en el estudio de Baltodano et al. (2016) se encontró que los adolescentes presentaron una actitud desfavorable en un $73.9 \%$ respecto al consumo de alimentos saludables. Los investigadores enfatizaron la importancia de adoptar una actitud adecuada o correcta cuando se refiere a temas sobre alimentación y nutrición saludable, ya que esta lleva a tener buenas prácticas y gozar de una calidad de vida. Además, recomendaron que las escuelas deben contar con métodos y estrategias suficientes para brindar adecuada educación nutricional.

Tabla 3

Nivel de Actitudes sobre alimentación saludable.

\begin{tabular}{lll}
$\begin{array}{l}\text { Actitudes sobre alimentación } \\
\text { saludable }\end{array}$ & $\mathrm{N}$ & $\%$ \\
Desfavorable & 98 & 16.6 \\
Favorable & 491 & 83.4 \\
Total & 589 & 100.0 \\
\hline
\end{tabular}

Como se puede observar en la tabla 4, la mayor parte de los estudiantes encuestados manifiesta una inadecuada práctica alimentaria. Estos hallazgos coinciden con los resultados de Delbino (2013), quien encontró que el $60 \%$ de los encuestados tiene malas prácticas alimentarias lo que es concomitante con Suarez (2014), quien evidenció que el $57 \%$ de la población de estudio presentó prácticas alimentarias inadecuadas. Sin embargo, estos resultados difieren con los de Bin et al. (2017), quien asevera que el $62.4 \%$ de los estudiantes presento prácticas alimentarias adecuadas. Hasta este punto, es vital una orientación nutricional basada en la realidad del estudiante, lo cual abarcaría tanto el ámbito educativo, social como el cultural. Además, se necesita un monitoreo en educación nutricional por parte de un especialista.
Por otro lado, en nuestro estudio se evidenció que solo el $46.7 \%$ de los adolescentes encuestados refiere haber consumido las tres comidas del día (desayuno, almuerzo y cena); no obstante, se encontró que un $42 \%$ consume comida rápida o alimentos inadecuados (hamburguesa, pollo broaster, pizza, chocolates, galletas, chupetines o postres queques, bizcochos, tortas), lo que indica un desorden alimenticio que traerá secuelas a futuro. Meléndez et al. (2012), coincidieron con nuestros resultados, debido a que, en un estudio realizado en México, encontraron que el $41 \%$ de los adolescentes encuestados refiere haber consumido comidas rápidas como las que se mencionaron anteriormente. Aunque su resultado, en cuanto al consumo de las tres comidas del día difiere, ya que el $64 \%$ de los adolescentes reportó haber consumido desayuno, almuerzo y cena. 
Conocimientos, actitudes y prácticas sobre una alimentación saludable en adolescentes de dos Instituciones Educativas Públicas, Lima Este-2018

Tabla 4

Nivel de Prácticas sobre alimentación saludable.

\begin{tabular}{lll}
$\begin{array}{ll}\text { Practicas sobre alimentación } \\
\text { saludable }\end{array}$ & $\mathrm{N}$ & $\%$ \\
\hline Inadecuado & 461 & 78.2 \\
Adecuado & 128 & 21.8 \\
Total & 589 & 100.0 \\
\hline
\end{tabular}

\section{CONCLUSIÓN}

Según los resultados obtenidos en el estudio realizado, se observó que más de la mitad de los adolescentes tiene un nivel de conocimiento regular, lo cual se pudo reflejar claramente en las preguntas no acertadas en cuanto al conocimiento sobre la cantidad de porciones de frutas y verduras que se debe consumir al día; asimismo, presentaron dificultades para contestar las preguntas sobre qué alimentos contienen mayor cantidad de proteína, fibra, azúcar refinada y sal. Además, desconocían los problemas de salud que pueden provocar una alimentación inadecuada.

Por otro lado, se encontró que los adolescentes presentaron una actitud favorable o positiva en optar por una alimentación saludable, pero sus patrones alimenticios los conllevan a consumir o preferir alimentos pocos saludables. Se patentiza que no siempre las actitudes positivas que los adolescentes tienen son determinantes al momento de elegir un alimento saludable.

En suma, a pesar de que la mayor parte de los estudiantes demostró una actitud favorable en cuanto a la alimentación saludable; sin embargo, tal proporción no la practica adecuadamente.

\section{Declaración de financiamiento y de conflictos de interés:}

El estudio fue financiado por los autores, quienes declaran no tener conflictos de interés.

\section{Correspondencia}

Yanina Fustamante Ticlla

Correo electrónico:

yaninafustamante@upeu.edu.pe
Giovanna Mirian Quispe Nuñoncca

Correo electrónico:

giovannaquispe@upeu.edu.pe

\section{REFERENCIAS BIBLIOGRÁFICAS}

Baltodano, G., Duarte, F y Alvertina, F. (2016). Conocimientos y actitudes sobre alimentación y nutrición que poseen los estudiantes de cuarto año del colegio privado bautista central y el colegio público Maestro Gabriel ubicados en la ciudad de Managua octubre 2015 (Tesis De Licenciatura, Universidad Nacional Autonoma De Nicaragua) Recuperado de http://repositorio. unan.edu.ni/4584/

Bin Shaziman S, Mohd Rani M, Nor Aripin K, abdul Hamid N, Wan Sulaim W, Yunus Abdullah R, (2017). Assessing nutritional knowledge, attitudes and practices and body mass index of adolescent residents of orphanage institutions in Selangor and Malacca. Pakistan Journal Nutrition, 16(6), 406-11. Recuperado de https://scialert.net/ abstract/?doi=pjn.2017.406.411

Cruz, D. (2015). Hábitos y conocimientos alimentarios que influyen en el estado nutricional de los alumnos de la escuela Eloy Alfaro de la parroquia de Montalvo durante Enero - Junio 2014. (Tesis de licenciatura, Universidad Técnica De Ambato). Recuperado de http://repositorio.uta.edu.ec/ bitstream/123456789/9488/1/DARIO\%20CRUZ. pdf

Delbino, C. (2013). Conocimientos, actitudes y prácticas alimentarias en adolescentes concurrentes al colegio F.A.S.T.A. (tesis licenciatura, Universidad F.A.S.T.A. Argentina) Recuperado de http://redi.ufasta.edu.ar:8080/ xmlui/bitstream/handle/123456789/81/304. pdf?sequence $=1$

Hernández R, Fernández C. y Baptista M. (2010). Metodología de la investigación. México: 5ta Ed.; Recuperado de https://www.esup.edu.pe/ descargas/dep_investigacion/Metodologia $\% 20$ de $\% 201$ \% $\% 20$ investigaci\%C3\%B3n $\% 205$ ta $\% 20$ Edici\%C3\%B3n.pdf 
Mondalgo J. (2016). Efectividad de un programa educativo en el nivel de conocimiento sobre alimentación saludable en los adolescentes de una Institución Educativa. (Tesis de licenciatura. Universidad Nacional Mayor De San Marcos). Recuperado de http://cybertesis.unmsm.edu.pe/ bitstream/handle/cybertesis/4776/Mondalgo lj.pdf;jsessionid=A493D05A4723BB1B1A6F061 8507B807C?sequence $=1$

Suarez C, Urrea L,Maldonado Y, Quintero Y ,(2014). Conocimientos, Actitudes y Prácticas sobre alimntacion y nutricion de loa adolescentes de 4 y 5 año de educacion media diversificada del municipio libertador del estado Merida, Venezuela. Revista MedULA p. 123-9. Recuperado de https://dialnet.unirioja.es/servlet/ articulo? codigo $=6439600$.

Donayre M. (2016). Publicidad televisiva alimentaria asociada al consumo de alimentos no saludables y sus motivos en escolares de nivel primario en una institución educativa privada - Los Olivos. (Tesis de licenciatura, Universidad Nacional Mayor De San Marcos). Recuperado de http:// cybertesis.unmsm.edu.pe/bitstream/handle/ cybertesis/6186/Donayre_tm.pdf?sequence $=1$

Rodrigues Leite, M., Santos Barbosa A, Góes da Silva D, Falcão Raposo O,Mendes-Netto R (2016). Conocimiento sobre alimentación y nutrición después del desarrollo de actividades de educación alimentaria entre niños y adolescentes deportistas. Pensar a Prática. 19(1):56-67. Recuperado de: https://www.researchgate. net/publication/318765692_conocimiento_ sobre_alimentacion_y_nutricion_despues_del_ desarrollo_de_actividades_de_educacion_ alimentaria entre ninos_y adolescentes deportistas

MelendezM,CañezG,Frias H. (2012). Comportamiento alimentario durante la adolescencia. ¿Nueva relación con la alimentación y el cuerpo? Re Mex Investig en Psicol, 4(2003, 99-111. Recuperado de: https://www.medigraphic.com/ pdfs/revmexinvpsi/mip-2012/mipM121j.pdf

Al-Yateem N, Rossiter R (2017). Nutritional knowledge and habits of adolescents aged 9 to 13 years in Sharjah, United Arab Emirates: a crosssectional study. East Mediterranean Health Journal. 2017;23(8):551-8. Recuperado de http://applications.emro.who.int/emhj/v23/08/ EMHJ_2017_23_08_551_558.pdf?ua=1\&ua=1

Abraham S, Noriega B, Shin J (2017). College students eating habits and knowledge of nutritional requirements. J Nutr Hum Health; 2(1), 13-7. Recuperado de http://www.alliedacademies.org/ abstract/college-students-eating-habits-andknowledge-of-nutritional-requirements-9188.html

Recibido: 20/01/19 Aceptado: 04/04/19 